\title{
Age- and Risk-Targeted Control of Schistosomiasis-Associated Morbidity Among Children and Adult Age Groups
}

\author{
David Gurarie ${ }^{1, *}$ and Charles H. King ${ }^{2}$ \\ ${ }^{I}$ Department of Mathematics and ${ }^{2}$ Center for Global Health and Diseases, Case Western Reserve University, Cleveland, \\ Ohio USA
}

\begin{abstract}
For helminth parasites, selection of optimal chemotherapy-based control of infection-related morbidity can take cues from several perspectives. One can focus on limiting intensity of infection or its spread within the community at any given time, and ask for efficient interventions to reduce either type of infectious burden. Alternatively, one can look at the long-term effects of infection and ask for control strategies aimed only at preventing long-term morbidity through structured treatments that vary by age. The latter approach is currently favored for schistosomiasis, where acute infection per $s e$ is believed to be less detrimental to health than the cumulative damage caused by long-term heavy infection. Here, we extend earlier approaches to modeling control of late-onset morbidity using age-stratified interventions, and then additionally consider population heterogeneity in terms of subgroups at high and low risk for development of chronic disease. We study the long-term effect age-structured mass therapy applied to the whole population $v s$. varying coverage for different population age strata. Given different subgroup risks for chronic disease, we also examine the possible utility of pretreatment risk screening towards optimal allocation of treatment resources.
\end{abstract}

Key Words: Schistosomiasis infection, morbidity and control, drug chemotherapy/supply and distribution, praziquantel, mathematical modeling.

\section{INTRODUCTION}

International agencies, including the World Health Organization (WHO), the Partnership for Child Development, and the Schistosomiasis Control Initiative currently advocate control of disease due to schistosomiasis by means of periodic administration of the antischistosomal drug, praziquantel, to affected populations [1-3]. Their general recommendation is to focus on school-age children, as this group is understood to have the greatest risk for both heavy schistosome infection and its consequent disease related sequelae [4-8]. Large-scale national control programs based on school-age drug treatment have now started or will soon be implemented in a number of sub-Saharan countries. The important question remains: Given the features of schistosome infection and its related disease formation, as well as the typical feature of program participation, what is the optimal timing for drug delivery that will minimize community burden of disease? In particular, how can we optimize the effect of population based control program in a resource-limited setting?

Schistosomiasis-related disease occurs in both acute and chronic forms, with some forms directly related to current granulomatous inflammatory response to parasite eggs trapped in host tissues, and other chronic forms developing as a consequence of cumulative tissues damage and scar

\footnotetext{
*Addres correspondence to this author at the Department of Mathematics Case Western Reserve University, Cleveland, Ohio USA;

E-mail: dxg5@case.edu.
}

formation $[9,10]$. In addition to these temporal factors, human age-related factors play an important role in schistosome ecology, as they strongly influence the process of both human-to-snail and snail-to-human transmission [11-13]. The age related effects result from age-specific differences in water use habits and frequency of water contact. The net result is a wide variation in risk of new infection and in snail contamination per contact. The development of anti-parasite protective immunity is also likely to be age-dependent. In 1996, Medley and Bundy [14] considered, on a preliminary basis, a dynamic model to optimize a single treatment in a stationary age-stratified population. Their group also developed programs to stimulate the cost and impact of multiple treatment given to an endemic community over a period of decades [5, 15-17]. Since this original analysis, new data have emerged on the heterogeneity of individual risk for severe schistosomiasis-related disease [18-21], and on the reversibility disease with treatment [22-25], and on longterm impact of repeated school-based therapy on the late outcomes of disease [26] and on local transmission of the infection $[27,28]$. The present paper revisits the optimal choice of drug control strategies given these new considerations.

\section{MATERIALS AND METHODS MODEL BACK- GROUND}

The role of age in schistosome transmission is associated with behavioral risk factors-- that is, age-variant water contact rates, resulting water contamination, and the mutual 
process of infection of snails and humans[13, 29-31]. For humans, both age and exposure history can also confer some level of protective immunity against reinfection and modulation of subsequent disease risk $[32,33]$.

On a basic scale, the chronic schistosomiasis pathologies develop from repeated accumulation and destruction of worm eggs in body tissues (liver, intestines or urinary tract), and through scarring caused by anti-inflammatory immune responses $[9,10,34]$. But this process can be modulated by individual genetic or age/exposure-related factors which increase or decrease the risk of developing severe chronic disease, as documented in recent studies [18-20].

To address the issues of infection, disease and possible effect of treatment interventions (e.g. mass drug therapy) Medley \& Bundy [14] proposed a mathematical model of age-structured population (over a life-span $a, 0<a<L$ ), with each age-group carrying its specific mean worm burden $\{w(a)\}$ (see also $[5-7,15,35])$. The chronic morbidity measured by age-specific tissue damage, $D(a)$ (e.g. fibrosis) develops gradually from accumulated host infection history $w(a)$, and is resolved at some rate $v$. So removing the source of inflammation $w$ one can expect a partial remediation of the "damage factor", $D$.

Let us note that, unlike either infection intensity (e.g., worm burden $w$, or the resulting excreted egg-count) or infection prevalence, the chronic damage factor is much harder to measure and quantify on population level. So, variable $D$ here serves as a crude proxy of the complex set of functional/ tissue pathologies that develop from schistosome egginduced inflammation.

Within such framework one can formulate dynamic model for time-age dependent variables $\{w(a, t) ; D(a, t)\}$ as coupled system of partial differential equations,

$$
\begin{aligned}
& \partial_{t} w(a, t)+\partial_{a} w(a, t)=\Lambda-\gamma w(a, t) \\
& \partial_{t} D(a, t)+\partial_{a} D(a, t)=\rho w(a, t)-v(D(a, t))
\end{aligned}
$$

Here $\Lambda$ denotes per-capita force of infection, $\gamma$ - natural worm attrition (death rate) in human hosts, and coefficients $\rho, v$ measure the rates of damage accretion (proportional to burden $w$ ) and its resolution. Rather than fixing resolution rate (linear function $v(D)$ ), Medley and Bundy's formulation [14] allowed a $D$-dependent rate $v(D)=v_{0} e^{-\sigma D} D$, so that increased damage $D$ would slow down the resolution (healing) process.

This model formulation allowed the authors to study the effect of age-structured chemotherapy, on infection levels $\{w(a)\}$ and the resulting morbidity (damage, $\{D(a)\}$ ). The chemotherapy can be accommodated in equation (1) by augmenting the natural worm mortality $\gamma$ by additional "drug-clearing" terms, represented here by a Dirac deltafunction

$$
\begin{aligned}
& \gamma \rightarrow \gamma+p \delta\left(a-a_{1}\right), \text { or } \\
& \gamma+p \sum_{j=1,2, \ldots} \delta\left(a-a_{j}\right)
\end{aligned}
$$

- summing over treatment ages $\left\{a_{1}<a_{2}<\ldots\right\}$. The choice of a Dirac (impulse) form for a single treatment session indicates its relatively short duration (compared to a human lifespan), but also a significant clearing (deworming) effect. Parameter $p=-\log (1-r)$ measures the efficacy of treatment, where $r$ is the fraction of killed worms per session. The treatment pattern (2) assumes mass drug therapy applied to the entire age-group. Alternatively, one can think of system (1)-(2) as representing a single host-history, or a "cohort-history" for nearly identical hosts.

Medley and Bundy proceeded to solve the model equations by fixing force of infection $\Lambda(a)$, using estimates based on field data, and solving differential equations (1), for the treated $\operatorname{system}(2)$, to find distributions $\{w(a), D(a)\}$, and explore the impact of treatment. In that case (prescribed force of infection $\Lambda$ ), Equations (1)-(2) allowed exact analytic solutions.

\section{Limitations of Established Models}

These earlier models make many simplifying assumption, which include an imposed (fixed) force of infection $\Lambda(a)$, and a homogeneous human population, both in terms of transmission behavior and the individual human susceptibility to chronic disease. In reality, a closed community would accumulate infection and chronic disease depending on local transmission environment (snail populations, the nature and frequency of contacts, etc.). So the force of infection $\Lambda$ in (1) should depend on dynamic variable $w(a, t)$ (in fact, on coupled human-snail system) rather than having single prescribed value [8].

The homogeneity assumption about the human population is also an oversimplification. Here possible dispersion of burden-levels and/or contact rates (within identical agegroups) may be less significant for transmission (infection force $\Lambda$ ), but not so for the development of chronic disease. Indeed, the levels of chronic morbidity could vary widely under similar infection patterns (see [18-21]).

\section{EXTENDED MODEL FORMULATION}

For the present analysis, we develop and extend the ideas of Medley and Bundy [14] and colleagues [5, 15-17], to accommodate variation in human-snail interactions within a closed community and allow some level of heterogeneity in chronic disease formation. Namely, we stratify population into "high" and "low" risk groups for disease.

We formulate the extended model, and explore several control strategies to predict late-term outcomes of treatment. Unlike [14], our models have no analytical solutions to work with. But we develop efficient numeric estimation procedures for program outcomes using Mathematica (Wolfram 
Research, Champaign IL), that allow similar analysis and prediction. Details of these programs are available from the corresponding author.

For our model, we adopt the following assumptions:

1. Human populations and environmental transmission factors are stationary

2. For individual humans, their infection (worm establishment) and water/snail contamination rates are agedependent.

3. Humans have either high or low risk for chronic disease (i.e. accumulation and resolution of egg-driven fibrosis) under an otherwise similar infection burden history. The low-risk individuals are able to resolve fibrosis at a faster rate compared to high-risk group: $v^{L} \gg v^{H}$

4. The risk factor for chronic disease is viewed as an innate host characteristic persisting throughout his/her life-span and independent of burden history. So all newborn are subdivided into two groups with population ratio $r^{H} / r^{L}$, and this ratio is maintained throughout all ages, i.e. $h_{a}{ }^{H} / h_{a}{ }^{L}=r^{H} / r^{L}$, for $0<a<L$, for high/low risk population fractions. This means, in essence, that neither schistosomiasis infection or chronic disease entail substantial rates of mortality [36].

5. Age-specific behavior and transmission patterns are identical for both (high / low risk) groups, so they carry the same mean worm burden: $w_{a}{ }^{H}=w_{a}{ }^{L}=w_{a}$. But their morbidity as measured by "damage" function $D$ will differ markedly.

In our approach we need to modify both the infection $(w)$ part of system (1), and the resulting evolution of morbidity. Namely, to account for human-snail interaction we make the force of infection $\Lambda$ dependent on communal mean worm burden $\{w(a)\}$, as well as age-dependent transmission (contact) parameters. [The derivation of $\Lambda(w(a))$ is outlined in the Appendix to this paper.]

We also replace the morbidity $(D)$ equation of system (1) with separate equations for the high and low-risk groups $\left\{D_{a}{ }^{H}, D_{a}^{L}\right\}$

$$
\begin{aligned}
& \partial_{a} w(a)=\Lambda(w)-\gamma w(a) ; \\
& \partial_{a} D^{L}(a)=\rho w(a)-v^{L}\left(D^{L}\right) ; \\
& \partial_{a} D^{H}(a)=\rho w(a)-v^{H}\left(D^{H}\right) ;
\end{aligned}
$$

In regard to disease resolution, we assume a linear decay rate for disease in the low-risk group, but adopt nonlinear pattern ([14]) for the high risk group,

$$
\begin{aligned}
& v^{L}(D)=v_{0} D ; \\
& v^{H}(D)=v_{1} e^{-\sigma D} D
\end{aligned}
$$

Thus, the initial "low" resolution rate of the high-risk group $v_{1}<v_{0}$, at the early stage of disease $(D=0)$, will further diminish as it accumulates damage ( $D>0$ ). Fig. (1) illustrates the burden distribution $w(a)$, and the ensuing morbidity patterns $\left\{D^{L}(a), D^{H}(a)\right\}$ for untreated population, and several selected values $v_{0}$ and $v_{1}$.
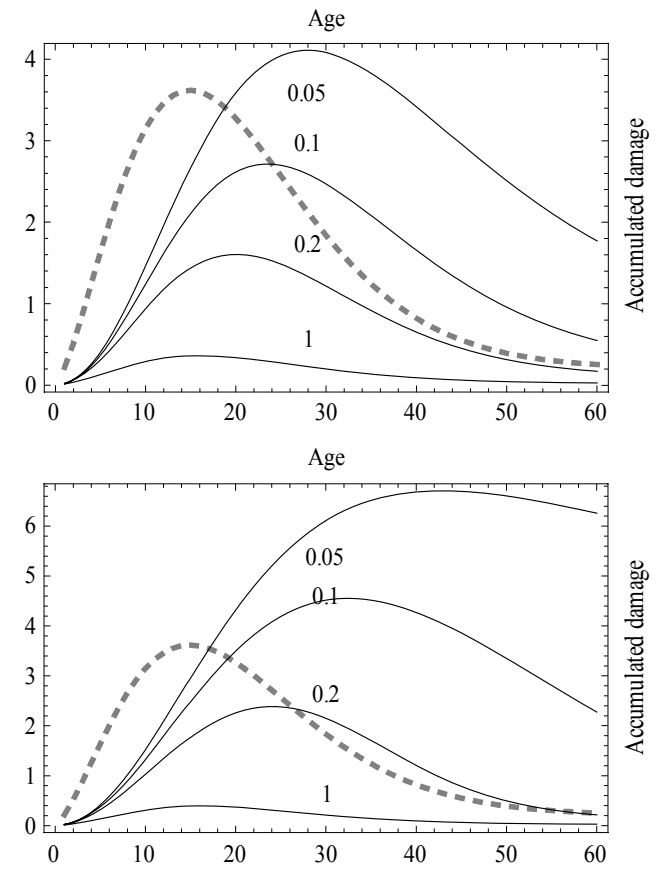

Fig. (1). Worm burden distribution (dashed) and the accumulated damage (solid line) at 4 different rates of disease resolution $\left\{v_{i}: i=0,1\right\}$ (in units $1 /$ years): top panel - for low-risk group, and bottom panel - for high-risk one.

To account for the age-patterned treatment of system (3)(4) we replace the natural worm mortality in the $w$ - equation with the treatment-enhanced one, as in (2). Another important consideration for any mass treatment program involves its fraction of coverage in the target population, as typically the program compliance levels, even for school-age groups, tends to be reduced on each subsequent round of therapy [37]. To this end, we need to extend the basic model (3), for the untreated community to encompass effects in a partially-covered one. We do it by further stratification of the entire population into treatment cohorts : "uncovered" - 0, "single treatment" - 1 , "twice repeated" -2 , etc.

We call the corresponding population coverage fractions: $f_{0}+f_{1}+\ldots+f_{m}=1$, with the subscript indicating the number of subsequent treatments. Clearly, each treatment cohort will carry a different burden level: $w^{k}=w^{k}(a) ; k=0,1, \ldots$ But the dynamic equations for all of them will be coupled through the common force of infection $\Lambda$, namely

$$
\left\{\begin{array}{l}
\partial_{a} w^{0}=\Lambda-\gamma^{0} w^{0} \\
\ldots \\
\partial_{a} w^{k}=\Lambda-\gamma^{k} w^{k} \\
\ldots
\end{array}\right.
$$


Here $\gamma^{0}$ indicates the natural worm mortality, and each $\gamma^{k}=\gamma^{0}+p \sum_{j=1}^{k} \delta\left(a-a_{j}\right)$ will add to it the outcome of $k$ consecutive treatments, as in Equation (2). All treatment cohorts share the common force of infection $\Lambda$, contributed by the entire community (see Appendix). Clearly, each treatment cohort will develop its own morbidity functions: $\left\{D^{k}=D^{k}(a): k=0,1, \ldots\right\}$ (for a hypothetical homogeneous community), or $\left\{D^{H, k}(a), D^{L, k}(a)\right\}$ - for heterogeneous (high / low risk) population.

\section{RESULTS}

Within the model formulation above, we can explore several treatment strategies involving realistic partial coverage and incomplete drug efficacy ( $\sim 85 \%$ deworming per session $[5,37])$. To do this, the entire population is subdivided into treatment cohorts as outlined above. N.B. the "risk factor" affects the morbidity-related treatment outcomes for persons in the high- or low-risk strata, but not their mean burden levels (we recall that "risk factor" in our context applies only to susceptibility to chronic disease, rather than infection risk).

\section{ANALYSIS OF CONTROL OPTIONS}

We consider two scenarios for mass treatment: one is based on blind selection of treatment cohorts, whereby each risk group is represented in proportion to its population size. The second scenario applies a hypothetical prescreening procedure (possibly genetic [18,19] or location-based [28]) to select high-risk individuals for more extensive treatment, which one hopes can bring down their morbidity levels to normal level (Fig. 1). We apply the following treatment protocols in the case of blind selection.

I. Three cohorts: (i) $60 \%$ of population treated at ages 6 and 12; (ii) $20 \%$ treated at age 6 only, (iii) remaining $20 \%$ untreated

II. In a typical field situation, compliance levels for multiple treatment sessions usually drops lower over time. Here we assume that $70 \%$ of those covered by the first treatment go on to receive as second one, $60 \%$ of those to a third one [37], hence treatment fractions

$$
\left\{f_{1}, f_{2}, f_{3}\right\}=\left\{.3\left(1-f_{0}\right), .28\left(1-f_{0}\right), .42\left(1-f_{0}\right)\right\}
$$

in terms of untreated fraction $f_{0}$. In addition, we allow a two-year gap between treatment sessions (based on WHO recommendations [38]) and let initial treatment age $a_{0}$ vary from 1 to 30 years.

In the second, (risk screening) scenario, the treated populations are subdivided among high/low risk groups based on their predicted risk of morbidity. We maintain the same overall treatment coverage rates as above, and hence, the same transmission levels and worm burden distributions. However, the screening is assumed to allow preferential se- lection of more of the high-risk hosts for treatment. We call this selected fraction $\phi$, and accordingly subdivide the highrisk group into 'treatment fractions' proportioned as $\{1-\phi, .3 \phi, .28 \phi, .42 \phi\} \quad$ (for $k=0 ; 1 ; 2 ; 3$ respectively). Parameter $\phi$ will be related to the efficacy $\psi$ of screening, (\% of positive selections), as

$$
\phi=\left(1-f_{0}\right)+f_{0} \psi
$$

in terms of untreated population fraction $f_{0}$. So zero efficacy implies $\phi=1-f_{0}$, as in (5), while $100 \%$ - efficient screening gives $\phi=1$.

In both cases, with and without risk screening, we ask for an optimal treatment age $a_{0}$ to attain the maximal reduction of late-age morbidity for high risk strata.

We fix resolution rates of morbidity for two risk groups as $v_{0}=.2$ /year (low risk), and $v_{1}=.05 e^{-.25 D}$ (high risk). As for damage accumulation rates we take: $\rho^{H}=1 ; \rho^{L}=.8$. The resulting worm burden $w$, and accumulated damage/morbidity $D^{L} ; D^{H}$, for individual cohorts, are shown in Fig. (2).

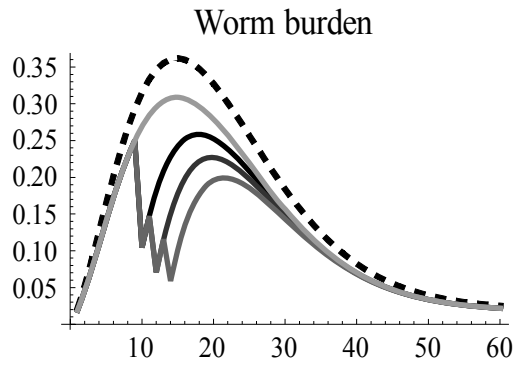

Low risk morbidity

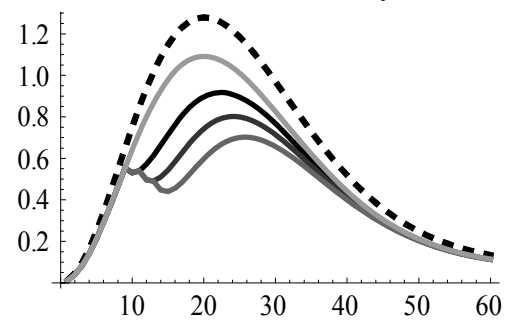

High risk morbidity

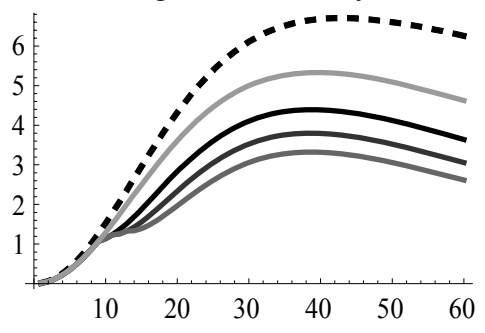

Fig. (2). Burden distribution (top) and accumulated damage/morbidity (middle, bottom) for 3 treatment cohorts of case I (shades of gray) vs. untreated population (dashed). The low and high risk groups differ by their resolution rates. 
The treatment ages are clearly marked as sharp drops of worm burden for the treated groups (top panel). Treatment renders long term benefit in the reduced $D$-levels (middle/ bottom panels of Fig. 2) for the treated cohorts. But the resulting (high/low) patterns are markedly distinct.

In both cases, we ask for an optimal timing of the initial treatment, $1<a_{0}<30$, to bring down the late-term mean (weighted) morbidity ${ }^{1}$ to its lowest value. Fig. (3) shows the results at two different coverage levels: $80 \%$ (i.e. $f_{0}=.2$ ) on the top, and $50 \%\left(f_{0}=.5\right)$ on the bottom.
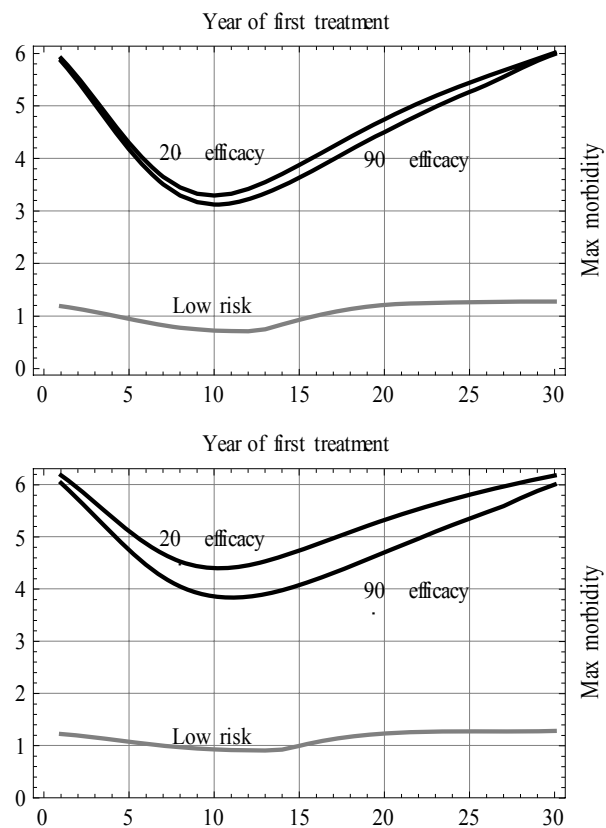

Fig. (3). Maximal late-term accumulated damage (cohort mean) as a function of the 'treatment age' $a_{0}$ in cases with and without risk screening $(70 \%, 60 \%$ - compliance), at two different drug coverage levels: $80 \%$ of eligible population (top), and $50 \%$ (bottom). Upper (black) curves on both plots are weighted high-risk groups, low (gray) curves are weighted low-risk ones. Two 'high risk' curves on each plot compare the results of selective screening, at two levels of its efficacy: $\psi=.9$ and $\psi=.2$.

In addition, we show the effect of selective screening of high/low sensitivity or effective detection, $\psi \quad(90 \%$ and $20 \%$ ), for detection of the high risk group. The optimal treatment age $a_{0}$ in all cases comes close to 10-12 years.

They also exhibit significant reduction of $\operatorname{Max}\left(D^{H}\right)$ compared to untreated levels, by factor $1 / 2$ for $80 \%$ treatment cover, and by $1 / 3$ - for $50 \%$ coverage. The effect of screening on $D^{H}$, however, diminishes with increased coverage (top and bottom black curves in Fig. 3).

${ }^{1}$ Each treatment cohort accumulates different maximal damage

$D^{k}(a): k=0,1, \ldots \quad$ (Figs. 2 and $\mathbf{4}$ ), and Fig. 3 shows its weighted mean

value: $\sum_{k=0,1 \ldots} f_{k} D^{k}$.
Next (Fig. 4), we show the burden and damage functions of the optimally treated populations (initial age $a_{0}=10$ ) with risk screening, vs. their expected values if left untreated.
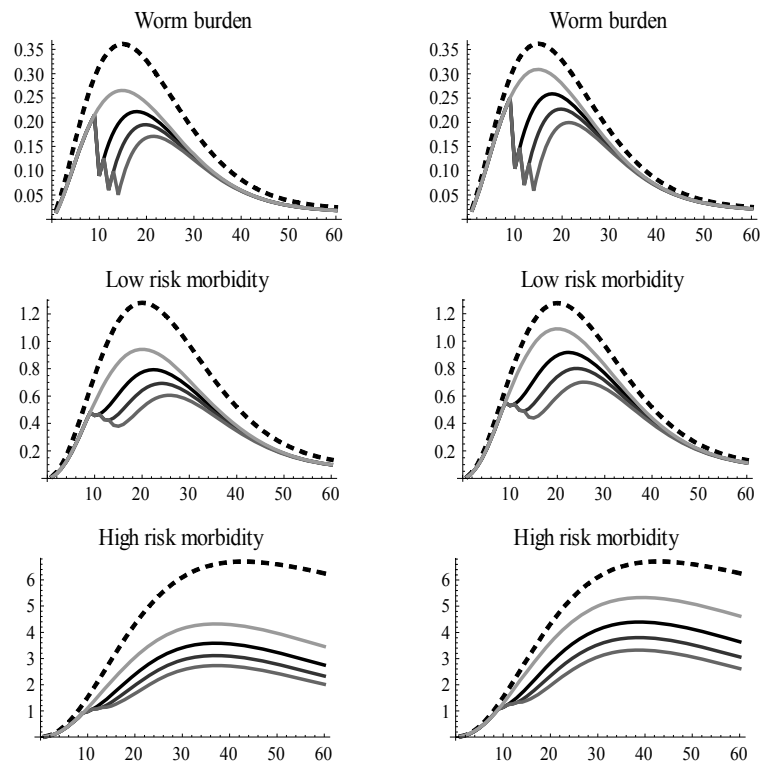

Fig. (4). Worm burden distribution by age (top) and accumulated morbidities of low-risk (middle) and high-risk (bottom) population cohorts, after 1 to 4 individual treatment rounds (shades of gray) in an optimally-treated, screened population $v s$. their expected distributions if left untreated (dashed lines), at two treatment coverage levels: $80 \%$ (left column) and 50\% (right column).

Finally, Fig. (5) demonstrates the weighted morbidity curves for high risk strata under optimal treatment of a riskscreened population.
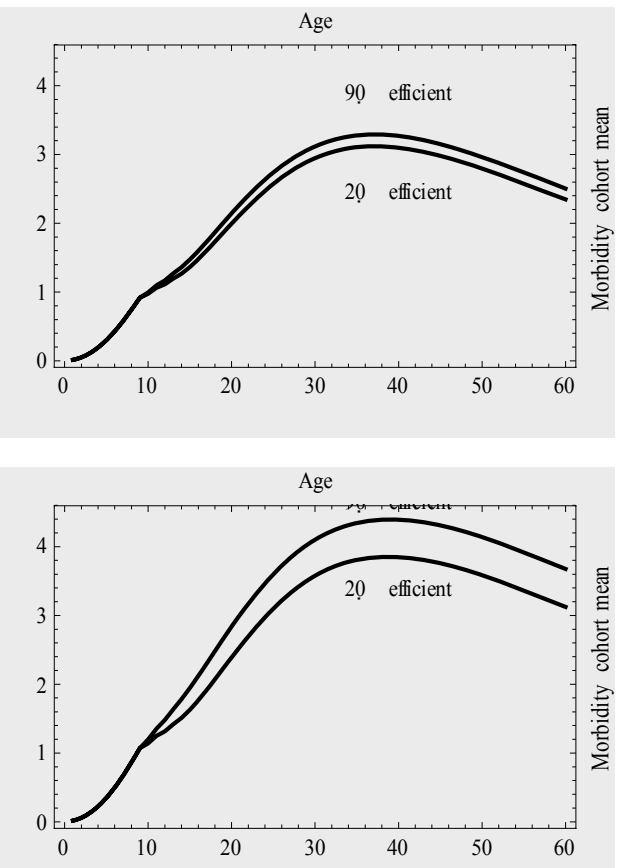

Fig. (5). Weighted morbidity curves for high risk strata under optimal treatment in a risk-screened population: $80 \%$ treatment cover (top) and 50\% (bottom) with two different levels of screening efficiency, $\Psi$. 
Once again the screening efficacy has negligible effect (over the entire life span) if drug treatment coverage is high, but screening grows in significance if coverage drops to lower levels.

\section{CONCLUSIONS}

Mathematical models can provide useful tools for analysis and prediction of long term effects of hypothetical control interventions in targeted settings. Often, this analysis would be difficult (or expensive) to assess completely using current evidence-based epidemiological approaches. In developing models for schistosome transmission and the ensuing morbidities of chronic schistosomiasis, we can apply them to hypothetical communities (homogeneous or heterogeneous) to explore different control strategies and identify the ones most likely to provide optimal benefits in terms of its efficacy and cost.

Our work extends some earlier studies in several ways. We have considered here the heterogeneity of populations in terms of morbidity risk, and examine the potential value of possible prescreening for morbidity risk for focused treatment efforts.

We find, among other things, that prescreening has minor effect (over an entire human life-span) when initial (childhood) drug treatment covers a large fraction of infected population. The value of screening grows in significance, however, when limited resources (or other considerations) ask for selective treatment, and a sizable fraction of infected people remain untreated.

As in any mathematical model, there are simplifying assumptions that go into the analysis, which may introduce quantities (variables) that are difficult to measure and quan- tify directly. Some variables, such as "mean worm burden" (or infection intensity) are reasonably justified for estimation of transmission, but burden impact may require more detailed analysis in terms of morbidity [18-21]. A possible link between "mean infection intensity" and "prevalence of morbidity" will depend on specific dispersal patterns of intensity levels within identical age/risk groups. Some commonly used distributions including Poisson and negative binomial, allow us to estimate connections between means and prevalences [39]. But these issues will require more fully refined mathematical models and tools - a plan for future work.

Here we did not specifically address the cost-effectiveness of different treatment strategies, or their optimal selection. Our analysis has focused on the optimal treatment age, and was meant to illustrate the utility of the method and model.

Let us note, however, that our model (and computer code) allows to explore a much wider range of optimization problems in health economics. For instance, given per capita cost of treatment and screening, and the known demographics (high- and low-risk population fractions), one can ask for an optimal allocation of resources (treatment coverage vs. screening coverage) to attain a desired result, i.e., reduction of the communal late-term morbidity, under different cost constraints. To this end, we can (as above) numerically sample a range of parameters and control strategies, then (i) estimate their communal outcomes in terms of morbidity reduction, (ii) estimate the associated incremental costs of more complex strategies. Such estimated values would allow one to formulate and solve the appropriate constrained optimization problem for health economic decision-making (cf. [8]).

\section{APPENDIX}

Here we derive the age-structured model for system (1) with the proper force of infection $\Lambda$, that is used in the analysis of chronic morbidity presented in this paper. It involves several essential parameters:

Table 1. Basic Variables and Parameters

\begin{tabular}{|c|c|}
\hline$\hat{\alpha}=\left(\alpha_{a}\right)$ & age dependent (worm) establishment rate \\
\hline$\hat{\beta}=\left(\beta_{a}\right)$ & age dependent (snail) contamination rate \\
\hline$\mu, \gamma$ & (natural) snail and worm mortalities \\
\hline$N$ & snail density \\
\hline$H ; h_{a}(0<a<L)$ & $\begin{array}{r}\text { total human population, and its age distribu- } \\
\text { tion: } \int_{0}^{L} h_{a} d a=1\end{array}$ \\
\hline
\end{tabular}

For numeric purposes we discretize continuous differential equations (3) for age bins $\{a=1,2, \ldots, P\}$ with time step $\Delta a$, and replace differential 'aging operator' $\partial_{a}$ with a $P \times P$ matrix 


$$
\mathbf{G}=\frac{1}{\Delta a}\left[\begin{array}{cccc}
1 & 0 & \cdots & 0 \\
-1 & 1 & 0 & \cdots \\
\cdots & \cdots & \cdots & \cdots \\
0 & \cdots & -1 & 1
\end{array}\right]
$$

The resulting algebraic system takes on the form

$$
\begin{aligned}
& (\mathbf{G}+\Gamma) \cdot \hat{w}=\hat{\Lambda} ; \\
& (\mathbf{G}+v \mathbf{I}) \cdot \hat{D}=\rho \hat{w}
\end{aligned}
$$

for vectors $\hat{w}=\left(w_{a}\right) ; \hat{h}=\left(h_{a}\right) ; \hat{\eta} ; \hat{\xi} ; \hat{D}$, etc. and worm attrition matrix $\Gamma=\gamma \mathbf{I}$ (natural, or therapy induced). The forces of infection: snail to human $\Lambda$ and human to snail $\lambda$ are given by

$$
\Lambda_{a}=\frac{\lambda_{H} N}{\lambda_{H}+\mu} \alpha_{a} ; \lambda_{H}=H \int \beta_{a} h_{a} w_{a} d a
$$

We take population pyramid (fractions) $\left\{h_{a}\right\}$ from UNFPA mortality/survival data for Africa (Fig. 6)
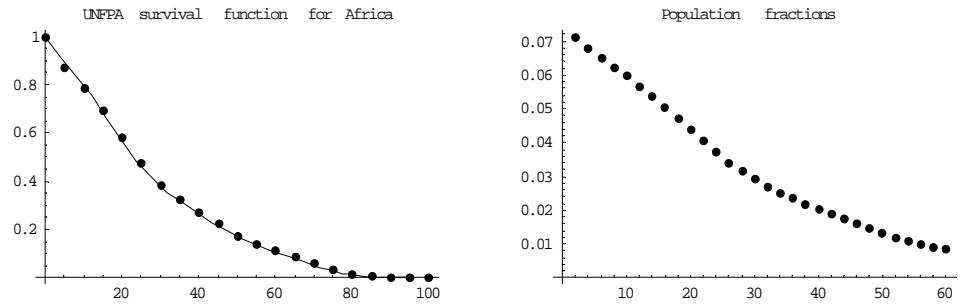

Fig. (6). Survival function (left), and 2-year age bin fractions (right) based on UNFPA census data for Africa (2000).

The establishment and contamination rates $\alpha_{a}, \beta_{a}$, adopted from [15], are given by functions: $A_{i} a e^{-B_{a} a^{2}}$ with suitable coefficients $A_{i}, B_{i}$ (Fig. 7).

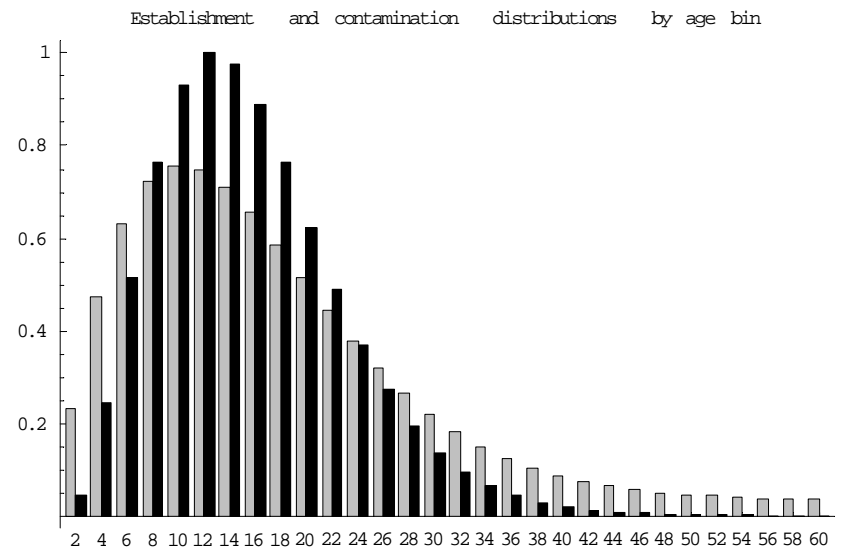

Fig. (7). Chan \& Bundy (1997) [15] establishment and contamination rates in schistosomiasis transmission (for 2-year age bins over $0<a<60)$.

We take typical worm attrition value $\gamma=.2 /$ year, and a range of values for (uncertain) morbidity resolution rate in the range: $.05<v<.2$, and allow (as Medley-Bundy [14]) both fixed $v$ (linear), and nonlinear morbidity resolution. Specifically,

$$
\begin{aligned}
& v^{L}=.2 / \text { year; (low risk) } \\
& v^{H}=v_{0} e^{-\sigma D} ; v_{0}=.05 / \text { year; } \\
& \sigma=.25 \text { (high risk) }
\end{aligned}
$$


So worm distribution $\hat{w}=\left(w_{a}\right)$ is proportional to the 'establishment vector' $\hat{\alpha}$, multiplied by 'inverse aging + attrition' matrix, $\Gamma=\gamma I$. A treatment would augment attrition $\Gamma \rightarrow \Gamma+\Theta$ with a diagonal treatment matrix $\operatorname{diag}\{\ldots, p, \ldots\}$, having nonzero entries $p=-\frac{\ln (1-r)}{\Delta a}$, at the 'treated' bins.

System (8) assumes complete drug coverage. For partial treatment the population is divided into treated/untreated cohorts (fractions $f_{1}, f_{2}=1-f_{1}$ ), with burden distributions $\hat{u} ; \hat{v}$, that obey

$$
\begin{aligned}
& (\mathbf{G}+\Gamma+\Theta) \cdot \hat{u}=\frac{\lambda_{H}(\hat{w}) N}{\lambda_{H}(\hat{w})+\mu} \hat{\alpha} ; \\
& (\mathbf{G}+\Gamma) \cdot \hat{v}=\frac{\lambda_{H}(\hat{w}) N}{\lambda_{H}(\hat{w})+\mu} \hat{\alpha}
\end{aligned}
$$

and mean distribution $\hat{w}=f_{1} \hat{u}+f_{2} \hat{v}$.

The resulting worm-burden distribution (vector) takes on the form

$$
\begin{aligned}
& \hat{w}=w_{0}(\mathbf{G}+\Gamma)^{-1} \cdot \hat{\alpha} ; \\
& w_{0}=N-\frac{\mu}{\lambda^{0}}
\end{aligned}
$$

where $\lambda^{0}$ encodes the basic parameters of Table 1 plus a possible treatment effect (increased worm attrition). In a similar fashion, partially covered cohorts have two different burden patterns: $\hat{u}$ - for treated fraction, and $\hat{v}$ - for untreated one, that obey

$$
\begin{aligned}
& \hat{u}=w_{0}(\mathbf{G}+\Gamma+\Theta)^{-1} \cdot \hat{\alpha} ; \\
& \hat{v}=w_{0}(\mathbf{G}+\Gamma)^{-1} \cdot \hat{\alpha} ;
\end{aligned}
$$

In all cases (8)-(12), solutions for $\hat{w}, \hat{u}, \hat{v}$ take on the form (13)-(14), with $w_{0}=N-\frac{\mu}{\lambda^{0}}$, and

$$
\lambda_{w}^{0}=H \widehat{h \beta} \cdot(\mathbf{G}+\Gamma)^{-1} \cdot \hat{\alpha}
$$

for untreated (or fully covered) population, or

$$
\begin{gathered}
\lambda_{u}^{0}=H \widehat{h \beta} \cdot(\mathbf{G}+\Gamma+\Theta)^{-1} \cdot \hat{\alpha} ; \\
\lambda_{v}^{0}=H \widehat{h \beta} \cdot(\mathbf{G}+\Gamma)^{-1} \cdot \hat{\alpha} \\
\lambda_{w}^{0}=f_{1} \lambda_{u}^{0}+f_{2} \lambda_{v}^{0}
\end{gathered}
$$

in case of partial coverage. Let us note that risk factor plays no role in establishing transmission levels and burden distribution, as in our assumption both (high/low) risk groups contribute equally. The difference appears in the ability to resolve accumulated damage. Thus we get the morbidity distribution

$$
\begin{aligned}
& \hat{D}^{L}=\rho_{L}\left(\mathbf{G}+v_{0}\right)^{-1} \cdot \hat{w} \\
& {\left[\mathbf{G}+v\left(\hat{D}^{H}\right)\right] \cdot \hat{D}^{H}=\rho \hat{w}}
\end{aligned}
$$

with $\hat{w}$ given by (13), or $\hat{w}=f_{1} \hat{u}+f_{2} \hat{v}$ (14) (partial coverage).

We non-dimensionalize system (8), or (12), and the resulting solutions (13)-(14) via rescaled quantities (marked with asterisk), and 'order of magnitude' parameters (subscript 0) 


\begin{tabular}{|c|c|}
\hline Establishment rates & $\hat{\alpha}=\alpha_{0} \hat{\alpha}^{*} ; \alpha_{0}=\operatorname{Max}\left(\alpha_{a}\right)$ \\
\hline Contamination rates & $\hat{\beta}=\beta_{0} \hat{\beta}^{*} ; \beta_{0}=\operatorname{Max}\left(\beta_{a}\right)$ \\
\hline Rescaled aging (in 'attrition' units $1 / \gamma)$ & $\mathbf{G}^{*}=\mathbf{G} / \gamma$ \\
\hline Dimensionless force of infection $\lambda^{0}$ & $\lambda^{*}=\left(\hat{h} \hat{\beta}^{*}\right) \cdot\left(\mathbf{I}+\mathbf{G}^{*}\right)^{-1} \cdot \hat{\alpha}^{*}$ \\
\hline
\end{tabular}

This yields the Basic Reproduction Number (BRN), $R_{0}=\frac{\alpha_{0} H \beta_{0} N}{\mu \gamma}$, and typical worm burden $\bar{w}_{0}=\frac{\mu}{H \beta_{0}}$, for the corresponding 'homogeneous' community (no age stratification). The rescaled burden solutions take on the form

$$
\begin{aligned}
& \hat{u}=\bar{w}_{0}\left(1-\frac{1}{R_{0} \lambda^{*}}\right)\left(\mathbf{G}^{*}+\Theta^{*}+\mathbf{I}\right)^{-1} \cdot \hat{\alpha}^{*} \\
& \hat{v}=\bar{w}_{0}\left(1-\frac{1}{R_{0} \lambda^{*}}\right)\left(\mathbf{G}^{*}+\mathbf{I}\right)^{-1} \cdot \hat{\alpha}^{*}
\end{aligned}
$$

where

$$
\begin{gathered}
\lambda_{w}^{*}=f \lambda_{1}^{*}+f_{2} \lambda_{v}^{*} ; \\
\lambda_{u}^{*}=\widehat{h \beta^{*}} \cdot\left(\mathbf{G}^{*}+\Theta^{*}+\mathbf{I}\right)^{-1} \cdot \hat{\alpha}^{*} ; \\
\lambda_{v}^{*}=\widehat{h \beta^{*}} \cdot\left(\mathbf{G}^{*}+\mathbf{I}\right)^{-1} \cdot \hat{\alpha}^{*}
\end{gathered}
$$

And the corresponding (low risk) morbidities $\hat{D}^{L}=\rho_{L}\left(\mathbf{G}^{*}+v_{0} \mathbf{I}\right)^{-1} \cdot\left(f_{1} \hat{u}+f_{2} \hat{v}\right)$.

For numeric simulations (implemented on Mathematica 5 and 6) we fix rescaled parameters $\left\{\alpha_{a}^{*}, \beta_{a}^{*}, h_{a}\right\}$ as in [15], take BRN $R_{0}=10$ (intense transmission environment), use the standard worm attrition $\gamma=.2 /$ year, vary morbidity $v_{0}$ in the range $.05 \leq v \leq .2$ / year, and allow two values $\sigma=0 ; .25$. While specific choice of parameters $v, \sigma$ may affect the late term morbidity patterns (Fig. 2), we found that percentage reduction curves (Fig. 3) exhibit less sensitivity to such variations. So our predictions of the efficacy of treatment will remain robust within fairly wide range of those parameters.

\section{ABBREVIATIONS AND VARIABLES}

WHO - World Health Organization

$L=$ Lifespan

$a=$ Age

$D=$ Cumulative infection-associated damage

$w=$ Individual (or communal mean) worm burden

$\Lambda=$ Per-capita force of infection

$\gamma=$ Natural worm attrition (death rate) in human hosts

$\rho=$ Rate of damage accretion (proportional to burden $w$ )

$v=$ Rate damage resolution

$p=$ Efficacy of treatment

$r=$ Fraction of killed worms per treatment session

\section{ACKNOWLEDGEMENTS}

This work was supported by NIH Research Grant \#R01TW008067 funded by the Fogarty International Center.

\section{REFERENCES}

[1] Chan MS, Nsowah-Nuamah NN, Adjei S, Wen ST, Hall A, Bundy DA. Predicting the impact of school-based treatment for urinary schistosomiasis given by the Ghana Partnership for Child Development. Trans R Soc Trop Med Hyg 1998; 92(4): 386-9.

[2] Fenwick A. New initiatives against Africa's worms. Trans R Soc Trop Med Hyg 2006; 100(3): 200-7.

[3] WHO. Preventive chemotherapy in human helminthiasis: Coordinated use of anthelminthic drugs in control interventions: a manual for health professionals and programme managers. Geneva: WHO Press 2006.

[4] Anderson RM, May RM. Infectious Diseases of Humans. Dynamics and Control. New York: Oxford University Press 1991.

[5] Chan MS, Guyatt HL, Bundy DA, Booth M, Fulford AJ, Medley GF. The development of an age structured model for schistosomiasis transmission dynamics and control and its validation for Schistosoma mansoni. Epidemiol Infect 1995; 115(2): 325-44.

[6] Chan MS, Guyatt HL, Bundy DA, Medley GF. Dynamic models of schistosomiasis morbidity. Am J Trop Med Hyg 1996; 55(1): 5262.

[7] Chan MS, Montresor A, Savioli L, Bundy DA. Planning chemotherapy based schistosomiasis control: validation of a mathematical model using data on Schistosoma haematobium from Pemba, Tanzania. Epidemiol Infect 1999; 123(3): 487-97. 
[8] Gurarie D, King CH. Heterogeneous model of schistosomiasis transmission and long-term control: the combined influence of spatial variation and age-dependent factors on optimal allocation of drug therapy. Parasitology 2005; 130(Pt 1): 49-65.

[9] Smith JH, Christie JD. The pathobiology of Schistosoma haematobium infection in humans. Hum Pathol 1986; 17: 333-45.

[10] King CL. Initiation and regulation of disease in schistosomiasis. In: Mahmoud AAF, ed. Schistosomiasis. London: Imperial College Press 2001: 213-64.

[11] Butterworth AE, Sturrock RF, Ouma JH, et al. Comparison of different chemotherapy strategies against Schistosoma mansoni in Machakos District, Kenya: effects on human infection and morbidity. Parasitology 1991; 103(Pt 3): 339-55.

[12] King $\mathrm{CH}$. Epidemiology of schistosomiasis: Determinants of transmission of infection. In: Mahmoud AAF, Ed. Schistosomiasis. London: Imperial College Press 2001: 115-32.

[13] Muchiri EM, Ouma JH, King CH. Dynamics and control of Schistosoma haematobium transmission in Kenya: an overview of the Msambweni Project. Am J Trop Med Hyg 1996; 55(5 Suppl): 12734.

[14] Medley GF, Bundy DA. Dynamic modeling of epidemiologic patterns of schistosomiasis morbidity. Am J Trop Med Hyg 1996; 55(5 Suppl): 149-58.

[15] Chan MS, Bundy DA. Modelling the dynamic effects of community chemotherapy on patterns of morbidity due to Schistosoma mansoni. Trans R Soc Trop Med Hyg 1997; 91(2): 216-20.

[16] Guyatt H, Evans D, Lengeler C, Tanner M. Controlling schistosomiasis: the cost-effectiveness of alternative delivery strategies. Health Policy Plan Dec 1994; 9: 385-95.

[17] Guyatt H. Different approaches to modelling the cost-effectiveness of schistosomiasis control. Mem Inst Oswaldo Cruz 1998; 93 (Suppl 1): 75-84.

[18] Dessein AJ, Hillaire D, Elwali NE, et al. Severe hepatic fibrosis in Schistosoma mansoni infection is controlled by a major locus that is closely linked to the interferon-gamma receptor gene. Am J Hum Genet 1999; 65(3): 709-21.

[19] Blanton RE, Salam EA, Ehsan A, King CH, Goddard KA. Schistosomal hepatic fibrosis and the interferon gamma receptor: a linkage analysis using single-nucleotide polymorphic markers. Eur J Hum Genet 2005; 13: 660-8.

[20] Wamachi AN, Mayadev JS, Mungai PL, et al. Increased ratio of tumor necrosis factor-alpha to interleukin-10 production is associated with Schistosoma haematobium-induced urinary-tract morbidity. J Infect Dis 2004; 190(11): 2020-30.

[21] Medhat A, et al. Increased interleukin-4 and interleukin-5 production in response to Schistosoma haematobium adult worm antigens correlates with lack of reinfection after treatment. J Infect Dis 1998; 178(2): 512-9.

[22] Hatz CF, Vennervald BJ, Nkulila T, et al. Evolution of Schistosoma haematobium-related pathology over 24 months after treatment with praziquantel among school children in southeastern Tanzania. Am J Trop Med Hyg 1998; 59(5): 775-81.

[23] King CH, Muchiri E, Ouma JH, Koech D. Chemotherapy-based control of schistosomiasis haematobia. IV. Impact of repeated annual chemotherapy on prevalence and intensity of Schistosoma haematobium infection in an endemic area of Kenya. Am J Trop Med Hyg 1991; 45(4): 498-508.

[24] Koukounari A, Fenwick A, Whawell S, et al. Morbidity indicators of Schistosoma mansoni: relationship between infection and ane- mia in Ugandan schoolchildren before and after praziquantel and albendazole chemotherapy. Am J Trop Med Hyg 2006; 75(2): 27886.

[25] Koukounari A, Gabrielli AF, Toure S, et al. Schistosoma haematobium infection and morbidity before and after large-scale administration of praziquantel in Burkina Faso. J Infect Dis 2007; 196(5): 659-69.

[26] Ouma JH, King CH, Muchiri EM, et al. Late benefits 10-18 years after drug therapy for infection with Schistosoma haematobium in Kwale District, Coast Province, Kenya. Am J Trop Med Hyg 2005; 73: 359-64.

[27] Satayathum SA, Muchiri EM, Ouma JH, Whalen CC, King CH. Factors affecting infection or reinfection with Schistosoma haematobium in coastal Kenya: Survival analysis during a nine-year, school-based treatment program. Am J Trop Med Hyg 2006; 75: 83-92.

[28] Clennon JA, Mungai PL, Muchiri EM, King CH, Kitron U. Spatial and temporal variations in local transmission of Schistosoma haematobium in Msambweni, Kenya. Am J Trop Med Hyg 2006; 75(6): 1034-41.

[29] Abel L, Demenais F, Prata A, Souza AE, Dessein A. Evidence for the segregation of a major gene in human susceptibility/resistance to infection by Schistosoma mansoni. Am J Hum Genet 1991; 48: 959-70.

[30] Ouma JH. Transmission of Schistosoma mansoni in an endemic area of Kenya with special reference to the role of human defaecation behaviour and sanitary practices. [Ph. D.]. Liverpool: University of Liverpool; 1987.

[31] Nooman ZM, Hasan AH, Waheeb Y, et al. The epidemiology of schistosomiasis in Egypt: Ismailia governorate. Am J Trop Med Hyg 2000; 62(2 Suppl): 35-41.

[32] Butterworth AE, Dunne DW, Fulford AJ, Ouma JH, Sturrock RF. Immunity and morbidity in Schistosoma mansoni infection: quantitative aspects. Am J Trop Med Hyg 1996; 55(5 Suppl): 109-15.

[33] Hagan P, Blumenthal UJ, Dunne D, Simpson AJG, Wilkins HA. Human IgE, IgG4 and resistance to reinfection with Schistosoma haematobium. Nature 1991; 349: 243-5.

[34] Chen MG, Mott KE. Progress in assessment of morbidity due to Schistosoma haematobium infection. Trop Dis Bull 1989; 86: R136.

[35] Chan MS, Anderson RM, Medley GF, Bundy DA. Dynamic aspects of morbidity and acquired immunity in schistosomiasis control. Acta Trop 1996; 62(2): 105-17

[36] van der Werf MJ, de Vlas SJ, Brooker S, et al. Quantification of clinical morbidity associated with schistosome infection in subSaharan Africa. Acta Trop 2003; 86(2-3): 125-39.

[37] King CH, Muchiri EM, Ouma JH. Evidence against rapid emergence of praziquantel resistance in Schistosoma haematobium, Kenya. Emerg Infect Dis 2000; 6(6): 585-94.

[38] WHO. Prevention and control of schistosomiasis and soiltransmitted helminthiasis: Report of a WHO expert committee. Technical Report Series 912. Geneva: World Health Organization; 2002. Report No.: 912

[39] Alexander N, Moyeed R, Stander J. Spatial modelling of individual-level parasite counts using the negative binomial distribution. Biostatistics 2000; 1(4): 453-63. 